\title{
Glutamate transporter GLT-1 mediates N-acetylcysteine inhibition of cocaine reinstatement
}

\author{
Kathryn J. Reissner ${ }^{1,2}$, Cassandra D. Gipson ${ }^{1}$, Phuong K. Tran ${ }^{1}$, Lori A. Knackstedt ${ }^{3}$, \\ Michael D. Scofield ${ }^{1}$, and Peter W. Kalivas ${ }^{1}$ \\ ${ }^{1}$ Department of Neurosciences, Medical University of South Carolina, Charleston, SC, USA \\ ${ }^{2}$ Department of Psychology, University of North Carolina, Chapel Hill, NC, USA \\ ${ }^{3}$ Department of Psychology, University of Florida, Gainesville, FL, USA
}

\section{Abstract}

Both pre-clinical and clinical studies indicate that $\mathrm{N}$-acetylcysteine (NAC) may be useful in treating relapse to addictive drug use. Cocaine self-administration in rats reduces both cystineglutamate exchange and glutamate transport via GLT-1 in the nucleus accumbens, and NAC treatment normalizes these two glial processes critical for maintaining glutamate homeostasis. However, it is not known if one or both of these actions by NAC is needed to inhibit relapse to cocaine seeking. To determine whether the restoration of GLT-1 and/or cystine-glutamate exchange is required for NAC to inhibit cue-induced reinstatement of cocaine seeking, we utilized the rat self-administration/extinction/reinstatement model of cocaine relapse. Rats were pre-treated in the nucleus accumbens with vivo-morpholino anti-sense oligomers targeting either GLT-1 or $\mathrm{xCT}$ (catalytic subunit of the cystine-glutamate exchanger) overlapping with daily NAC administration during extinction (100 mg/kg, i.p. for the last 5 days). Rats then underwent cueinduced reinstatement of active lever pressing in the absence of NAC, to determine if preventing NAC-induced restoration of one or the other protein was sufficient to block the capacity of chronic NAC to inhibit reinstatement. The vivo-morpholino suppression of $\mathrm{xCT}$ reduced cystine-glutamate exchange but did not affect NAC-induced reduction of reinstated cocaine seeking. In contrast, suppressing NAC-induced restoration of GLT-1 not only prevented NAC from inhibiting reinstatement, but augmented the capacity of cues to reinstate cocaine seeking. We hypothesized that the increased reinstatement after inhibiting NAC induction of GLT-1 resulted from increased extracellular glutamate, and show that augmented reinstatement is prevented by blocking mGluR5. Restoring GLT-1, not cystine-glutamate exchange, is a key mechanism whereby daily NAC reduces cue-induced cocaine reinstatement.

\footnotetext{
(C) 2014 Society for the Study of Addiction

Correspondence to: Kathryn J. Reissner, Department of Psychology, University of North Carolina, Chapel Hill, NC 27599, USA. reissner@unc.edu.

Disclosure/Conflict of Interest

None.

Authors Contribution

KR designed and performed the majority of experiments and wrote the manuscript, together with PK. PK supervised experimental design and data analysis. LK and PK provided significant intellectual contribution to interpretation of results. PT assisted with selfadministration training and histological analysis. CG and LK assisted glutamate-uptake assays, and MS assisted with microscopy. All authors have critically reviewed content and approved final version submitted for publication.
} 


\section{Keywords}

Cocaine; cystine-glutamate exchange; glutamate; glutamate transport; mGluR5; nucleus accumbens; $\mathrm{N}$-acetylcysteine; reinstatement

\section{INTRODUCTION}

Considerable evidence indicates that chronic cocaine abuse leads to an enduring disruption of glutamate homeostasis and glutamatergic signaling within the nucleus accumbens, including suppression of both the cystine-glutamate exchanger and high affinity glutamate transporters (Kalivas 2009; Wolf \& Ferrario 2011). N-acetylcysteine (NAC) is a cystine prodrug that activates the cystine-glutamate exchanger $\left(\mathrm{xc}^{-}\right)$, a key process for maintaining extrasynaptic glutamate concentrations (Millea 2009; Lewerenz et al. 2013). Chronic NAC reverses chronic cocaine-induced glutamate dysregulation, including normalizing drugdependent decreases in extracellular glutamate levels, and protein expression levels of $\mathrm{xCT}$ (the catalytic subunit of the cystine-glutamate exchanger) and GLT-1 (a high affinity astroglial glutamate transporter) (Baker et al. 2003; Knackstedt, Melendez \& Kalivas 2010). Extensive pre-clinical data supports the therapeutic potential for NAC in addiction and other psychiatric conditions (Dean, Giorlando \& Berk 2011; Olive et al. 2012), and clinical data provides promising findings for treating cocaine and marijuana addiction (Kalivas \& Volkow 2011; Schmaal et al. 2012; Gray et al. 2012). Hence, understanding the mechanism of action of NAC may aid in discovering therapeutic targets for treating addiction.

While it is clear that chronic NAC affects glutamate homeostasis by promoting both cystineglutamate exchange and GLT-1, it is not understood whether one or both of these actions is required for NAC to inhibit the reinstatement of cocaine seeking. Correspondingly, it is unclear whether the putative therapeutic effect of NAC is mediated primarily by its action on XCT or GLT-1, or both. Here, we used an antisense vivo-morpholino strategy to inhibit the capacity of NAC to induce either cystine-glutamate exchange or GLT-1, and tested animals for cocaine seeking 1 day after the last NAC treatment, to isolate a chronic versus acute effect. We found that restoring GLT-1, not cystine-glutamate exchange, was critical for chronic NAC to inhibit cue-induced reinstatement of cocaine seeking.

\section{MATERIALS AND METHODS}

\section{Animal surgeries and behavioral training}

Male Sprague Dawley rats (Charles River, 300-350 g; Raleigh, NC, USA) were housed on a 12-hour reverse-light cycle and provided limited chow (20 g per day) and water ad libitum. All animal use practices were per National Institutes of Health guidelines for laboratory animal care and were approved by the Medical University of South Carolina Institutional Animal Care and Use Committee. For surgical implantation of intrajugular catheters and intracranial cannulas, rats were anesthetized with ketamine $\mathrm{HCl}(100 \mathrm{mg} / \mathrm{kg}$, i.m.) and xylazine ( $7 \mathrm{mg} / \mathrm{kg}$, i.m.). Ketorolac ( $3 \mathrm{mg} / \mathrm{kg}$, i.p.) was administered before surgery to provide analgesia. For catheter implantation, a guide cannula (C313G, Plastics One, Roanoke, VA, USA) attached to silastic tubing (.025 ID, .047 OD, Bio-sil; Fisher Scientific, 
Hampton, New Hampshire) was inserted subcutaneously between the shoulder blades and exited the skin via a dermal biopsy hole $(3 \mathrm{~mm})$. Catheter exiting the skin was secured by a backpack (Instech Laboratories, Plymouth Meeting, PA, USA). The other end of the catheter was inserted $3 \mathrm{~cm}$ into the right jugular vein and then securely sutured to the underlying muscle tissue. Timentin antibiotic $(10 \mathrm{mg} / 0.1 \mathrm{ml}$, i.v. $)$ was infused at the end of the surgery. Prior to onset of self-administration, animals received one 12-hour food training session in which an active lever press resulted in the presentation of a chow pellet. Self-administration was performed 2 hours per day on a fixed-ratio schedule, in which in response to an active lever press, animals received an intravenous infusion of cocaine ( $0.2 \mathrm{mg}$ per infusion) paired with the presentation of light and tone and followed by a 20 -second time-out period. Criterion for self-administration was a minimum of 10 days in which rats received 10 or more daily cocaine infusions. Following self-administration, rats entered extinction training during which cocaine and drug-paired cues were no longer delivered in response to an active lever press. Only animals who had extinguished to below 65 presses per day at the onset of chronic morpholino and NAC treatments were included in the study. During the cue-induced reinstatement test, active lever presses were reinforced by the cocaine-associated cues but not cocaine.

\section{Vivo-morpholino and NAC treatment protocol}

Figure 1a shows the treatment protocol used: rats were treated daily for 3 days with control sequence or antisense vivo-morpholino beginning on day 10 of extinction training, and NAC $(100 \mathrm{mg} / \mathrm{kg}$, i.p.) or saline administered daily for 5 days beginning on day 12 of extinction. Control sequence was the exact same order of bases as the antisense, but in reverse sequence order. Microinjections of vivo-morpholinos were timed such that protein levels would be suppressed during NAC administration and the reinstatement session (Reissner et al. 2012). Animals were behaviorally tested or tissue harvested 24 hours after the last injection of NAC or saline. During extinction training, rats were micro-injected daily for 3 days with vivo-morpholinos 2 hours after the end of each extinction session. Starting on the third day of vivo-morpholino microinjection, rats were administered either saline or NAC (100 mg/kg, i.p.; Sigma-Aldrich, St. Louis, MO, USA) 2 hours prior to the extinction session. This dose and pre-treatment schedule were based on previous studies showing NAC efficacy in elevating both cystine-glutamate exchange and GLT-1, and inhibiting reinstated cocaine seeking (Knackstedt et al. 2010; Moussawi et al. 2011). NAC was prepared in $27 \mathrm{mg} / \mathrm{ml}$ $\mathrm{NaOH}$ in saline and adjusted to physiological $\mathrm{pH}$, then used immediately. Four more extinction sessions were conducted with NAC or saline pre-treatment without vivomorpholino pre-treatment. In a final experiment, animals were also pre-treated with saline or 3-[(2-methyl-4-thiazolyl)ethynyl]pyridine (MTEP; $0.5 \mathrm{mg} / \mathrm{kg}$, i.p.; Tocris Bioscience, Minneapolis, MN, USA) 10 minutes prior to beginning a cue-induced reinstatement session. This dose of MTEP was based on our previous dose-response indicating that $0.5 \mathrm{mg} / \mathrm{kg}$ was a maximum dose that would not inhibit cue-induced cocaine reinstatement (Wang et al. 2013).

\section{Microinjections and histology}

The microinjection procedure and vivo-morpholino sequences (Gene Tools, Inc., Philomath, OR, USA) were exactly as previously described (Reissner et al. 2012, 2013). All vivo- 
morpholinos were diluted 1:50 in sterile phosphate-buffered saline and a total volume of $1 \mu \mathrm{l}$ was injected per side (30 pmol per injection). A higher dose was used in initial studies; because reinstatement for these animals was not significantly different with lower doses, data were pooled. Coordinates used for the nucleus accumbens were $+1.5 \mathrm{~A} / \mathrm{P},+1.8 \mathrm{M} / \mathrm{L}$, $-5.5 \mathrm{D} / \mathrm{V}$, and microinjections were performed $2 \mathrm{~mm}$ below the canula base (Paxinos \& Watson 2005). An antisense vivo-morpholino was constructed for xCT and GLT-1 and control sequences for each were the same bases in reverse order. Histology to confirm cannula placement was performed following an overdose of pentobarbital and perfusion with saline. Brains were fixed in $4 \%$ formaldehyde and coronal slices $(100 \mu \mathrm{m})$ were made for Cresyl violet staining.

\section{${ }^{3} \mathrm{H}$-Glutamate uptake}

Sodium-dependent and sodium-independent uptake activity was measured using an in vitro slice preparation as described previously (Knackstedt et al. 2010). Briefly, drug-naïve rats were decapitated and bilateral punches of the nucleus accumbens core were dissected and sliced into $250 \times 250 \mu \mathrm{m}$ sections using a McIllwan tissue chopper (St. Louis, MO, USA). Slices were incubated at $37^{\circ} \mathrm{C}$ in either oxygenated Krebs-Ringer's solution phosphate buffer (140 mM NaCl, $1.3 \mathrm{mM} \mathrm{CaCl}_{2}, 1.2 \mathrm{mM} \mathrm{KH}_{2} \mathrm{PO}_{4}, 5 \mathrm{mM}$ HEPES, $10 \mathrm{mM}$ glucose and $1 \mathrm{mM} \mathrm{MgCl}_{2}, \mathrm{pH}$ 7.4) to quantify sodium-dependent uptake, or an identical buffer in which $\mathrm{NaCl}$ was replaced with $140 \mathrm{mM}$ choline chloride to measure sodium-independent uptake. Uptake was stimulated by the addition of L- $\left[{ }^{3} \mathrm{H}\right]$ glutamate $(150 \mathrm{nM}, 51 \mathrm{Ci} / \mathrm{mM}$; Perkin-Elmer, Boston, MA, USA) to slices in the presence of $10 \mu \mathrm{M} \mathrm{L}$-glutamate. This concentration was chosen based on our previous findings that uptake at this concentration is significantly decreased following cocaine self-administration (Knackstedt et al. 2010). The uptake reaction was conducted at $37^{\circ} \mathrm{C}$ for 15 minutes and then terminated by washing the slices in ice-cold, sodium-free Krebs-Ringers buffer. Slices were solubilized at room temperature with $1 \%$ sodium dodecyl sulfate (SDS) and the level of radioactivity determined using a liquid scintillation counter. Protein content was measured using a bicinchoninic acid assay protein assay (Thermo Scientific, Rockford, IL, USA) and the cpm/mg protein calculated.

\section{Western blotting}

For Western analysis, a membrane subfraction was performed as described previously (Reissner et al. 2011). All protein buffers were supplemented 1:100 with Halt protease and phosphatase inhibitors, including ethylene-diaminetetraacetic acid (Thermo Scientific, Rockford, IL, USA). Membrane pellet was resuspended in $30 \mu \mathrm{l} 1 \times$ RIPA buffer (Thermo Scientific) plus 1.0\% SDS and inhibitors. Equal microgram quantities were loaded per lane on a $10 \%$ Criterion Bis-Tris gel (Bio-Rad, Hercules, CA, USA). Following electrophoresis, proteins were transferred for 7 minutes on an iblot transfer apparatus (Life Technologies, Carlsbad, CA, USA), and blocking was performed for 1 hour at room temperature in $3.0 \%$ non-fat dry milk in tris buffered saline plus $0.1 \%$ tween-20. Primary antibodies against GLT-1 (Abcam, ab41621, 1:500; Cambridge, MA, USA), calnexin (Enzo Life Sciences, ADI-SPA-860, 1:1000; Farmingdale, NY, USA) were incubated overnight at $4^{\circ} \mathrm{C}$. Samples were heated at $37^{\circ} \mathrm{C}$ for 30 minutes prior to electrophoresis. 


\section{Statistics}

Glutamate uptake was evaluated using a Student's $t$-test, reinstatement data using a threeway ANOVA and Western blotting using a one-way ANOVA. For post hoc multiple comparisons either a Dunnett's test or a Bonferroni $t$-test were used.

\section{RESULTS}

Cocaine self-administration reduces both cystine-glutamate exchange and glutamate transport via GLT-1 in the nucleus accumbens (Baker et al. 2003; Sari et al. 2009; Knackstedt et al. 2010). We employed an antisense vivo-morpholino strategy to evaluate the role played by each protein in cue-induced reinstatement of cocaine seeking (Reissner $e t$ al. 2012). Figure 1a shows the treatment protocol used. Active lever pressing during selfadministration and extinction training, as well as average number of cocaine infusions taken during self-administration, were statistically equivalent between animals assigned to the saline versus NAC groups (not shown). A three-way ANOVA revealed a significant interaction between oligomer, i.p. treatment and time $\left(\mathrm{F}_{(1,100)}=9.389, P=0.003\right.$, Fig. 1). This was followed-up with a two-way ANOVA to analyze lever pressing during the last day of extinction, which revealed no effect of oligomer or i.p. treatment, nor any interaction. A subsequent two-way ANOVA to analyze reinstatement between groups indicated a significant interaction between oligomer and i.p. treatment (interaction $\mathrm{F}_{(1,100)}=9.078, P=$. 003). Figure $1 \mathrm{~b}$ shows that reinstatement was reduced by NAC treatment in animals treated with control sequence, even though the last NAC injection was administered 24 hours before the reinstatement session. Similarly, NAC reduced reinstatement in animals pre-treated with $\mathrm{xCT}$ vivo-morpholino antisense to inhibit the cystine-glutamate exchanger. In contrast, NAC treatment in animals administered antisense vivo-morpholino targeted to GLT-1 mRNA showed a marked potentiation of reinstated behavior. Importantly, treatment with antisense morpholino targeting either xCT or GLT-1 did not alter cue-induced reinstatement among rats treated with daily saline rather than NAC prior to reinstatement testing. Figure 1c shows the location of the tip of all microinjection cannula determined from Cresyl violet stained coronal sections, into the nucleus accumbens core.

The capacity of antisense vivo-morpholinos to reduce protein expression of GLT-1 and xCT has been previously reported (Reissner et al. 2012). However, because of the surprising lack of effect of $\mathrm{xCT}$ antisense on the behavioral effect of NAC, we sought to extend this finding to confirm that $\mathrm{xCT}$ antisense morpholinos functionally impair the cystine-glutamate exchanger. The effect of xCT antisense vivo-morpholino was quantified by measuring $\mathrm{Na}^{+}-$ dependent and -independent glutamate uptake ${ }^{3} \mathrm{H}$-glutamate in accumbens tissue slices (Bridges et al. 2012). Figure 2a shows that compared with the $\mathrm{xCT}$ control sequence, $\mathrm{xCT}$ antisense vivo-morpholino microinjections significantly reduced $\mathrm{Na}^{+}$-independent ${ }^{3} \mathrm{H}$ glutamate uptake by 23 percent $\left(\mathrm{t}_{(10)}=3.05, P=0.012\right)$, but did not affect $\mathrm{Na}^{+}$-dependent uptake that characterizes transport through glutamate transporters, predominantly GLT-1 (Danbolt 2001). This reduction in $\mathrm{Na}^{+}$-independent uptake is in agreement with the 25 percent reduction in $\mathrm{xCT}$ protein expression previously reported using the same $\mathrm{xCT}$ vivomorpholino antisense approach (Reissner et al. 2012), and confirms functional suppression by the $\mathrm{xCT}$ antisense, with lack of effect on GLT-1. It is worthwhile to note that while 
modest, this degree of suppression is also similar to the reported effect of cocaine selfadministration and extinction on $\mathrm{xCT}$ protein expression in the accumbens (Knackstedt et al. 2010).

Because reinstatement was so markedly affected by treatment with GLT-1 antisense plus NAC (Fig. 1b), we also examined membrane content of GLT-1 protein after both GLT-1 antisense vivo-morpholino and NAC. Figure $2 b$ shows that cocaine-experienced animals treated with control vivo-morpholino had reduced GLT-1 compared with yoked-saline control oligomer-treated saline animals, and that cocaine-reduced GLT-1 levels were restored by NAC treatment (black bar) $\left(\mathrm{F}_{(3,27)}=5.04, P=0.007\right)$. However, the capacity of NAC to elevate GLT-1 was abolished by treating the accumbens with GLT-1 antisense vivomorpholino (hatched bar).

The augmented cue-induced reinstatement following antisense vivo-morpholino suppression of GLT-1 when combined with NAC was surprising. NAC is known to elevate the basal levels of glutamate by stimulating the efflux of glutamate through the cystine-glutamate exchanger, and this is augmented by the reduced glutamate uptake produced by reduced GLT-1 activity (Baker et al. 2003; Melendez, Vuthiganon \& Kalivas 2005). Increased extracellular glutamate concentrations would thus have access to extrasynaptic glutamate receptors, including mGluR5, that strongly regulate cocaine reinstatement; stimulation potentiates while antagonism of mGluR5 inhibits reinstatement (Kumaresan et al. 2009; Martin-Fardon et al. 2009; Wang et al. 2013). Together, these data pose a hypothesis that concomitant increased extracellular glutamate via $\mathrm{xc}^{-}$and suppression of uptake leads to increased stimulation of mGluR5 which accounts for the potentiated reinstatement seen in animals treated with NAC plus GLT-1 antisense vivo-morpholino. To test this hypothesis, we paired a sub-threshold dose of mGluR5 negative allosteric modulator MTEP with GLT-1 antisense in the accumbens and systemic NAC treatment. Figure 3a shows that administration of a dose of MTEP $(0.5 \mathrm{mg} / \mathrm{kg}$, i.p. $)$ that is sub-threshold for inhibiting reinstatement in animals treated with GLT-1 antisense plus saline, abolished the potentiated reinstatement produced in rats treated with GLT-1 antisense plus NAC (NAC versus saline $\mathrm{F}_{(1,55)}=6.88, P=0.011 ;$ MTEP versus saline $\mathrm{F}_{(1,55)}=7.49, P=0.008$; interaction was not significant).

\section{DISCUSSION}

Our data indicate that restored expression of GLT-1, but not xCT, is critically important for the ability of NAC to suppress cue-primed reinstatement of cocaine seeking. Indeed, preventing the restoration of GLT-1 by NAC actually potentiated cue-induced reinstatement. The down-regulation of GLT-1 by antisense vivo-morpholino would be expected to reduce the elimination of extracellular glutamate and thereby increase extracellular levels of glutamate, akin to what occurs when GLT-1 is pharmacologically inhibited (Danbolt 2001). This increase would likely be exacerbated by NAC, which increases glutamate release by activating cystine-glutamate exchange (Baker et al. 2003; Moran et al. 2005; Bridges et al. 2012). The potential accumulation of glutamate in the extracellular space could result in the stimulation of extrasynaptic glutamate receptors, including mGluR5. Pharmacological activation of mGluR5 in the accumbens promotes cocaine reinstatement (Moussawi et al. 
2009; Wang et al. 2013), while inhibiting mGluR5 prevents reinstated cocaine seeking (Backstrom \& Hyytia 2007; Kumaresan et al. 2009; Wang et al. 2013). Accordingly, we found that the augmented reinstatement in NAC-treated animals produced by pre-treatment with GLT-1 antisense vivo-morpholino was prevented by administration of a negative allosteric modulator of mGluR5, MTEP.

How NAC increases GLT-1 and cystine-glutamate exchange is unclear. It was previously assumed that NAC was deacetylated, then dimerized to form cystine in the extracellular space and act as a substrate to drive cystine-glutamate exchange (Flanagan \& Meredith 1991). However, it was recently shown that NAC enters cells primarily via cysteine transporters (Kupchik et al. 2012). Moreover, in tissue slices, high concentrations of NAC provoke glutamate release via cystine-glutamate exchange that potentiates excitatory postsynaptic currents (EPSCs) in accumbens medium spiny cells. This potentiation is blocked by MTEP (Kupchik et al. 2012); thereby, providing a mechanistic link between NAC and mGluR5 stimulation that could mediate the potentiated reinstatement in rats treated with the combination of GLT-1 antisense vivo-morpholino and NAC.

A decrease in expression of GLT-1 in the accumbens is one of the most consistent findings across drugs of abuse and administration protocols found following self-administration of cocaine, heroin, alcohol and nicotine (Knackstedt et al. 2010; Fischer-Smith, Houston \& Rebec 2012; Sari \& Sreemantula 2012; Gipson et al. 2013). In addition, restored GLT-1 levels are observed following daily treatment with either NAC or ceftriaxone, two compounds that suppress reinstated cocaine, nicotine, alcohol, food and/or heroin seeking (Zhou \& Kalivas 2007; Sari et al. 2009, 2011; Knackstedt et al. 2010; Moussawi et al. 2011; Ramirez-Nino, D'Souza \& Markou 2013). NAC and ceftriaxone also restore cystineglutamate exchange in rats trained to self-administer cocaine (Trantham-Davidson et al. 2012). While we show that this latter action is not critical for inhibiting cocaine reinstatement, these data appear contrary to the assertions we and others have made regarding a pivotal role for cystine-glutamate exchange in reinstated drug seeking (Kalivas 2009; Bridges et al. 2012). However, it is important to distinguish studies that employ a single, acute NAC pre-treatment prior to reinstating cocaine seeking and studies such as ours that use daily NAC and test animals in the absence of an acute NAC pre-treatment. The inhibition of cocaine reinstatement by NAC has been directly linked to activation of cystineglutamate exchange to promote the release of non-synaptic glutamate, and thereby stimulate presynaptic mGluR2/3 to reduce synaptic glutamate release probability (Kalivas 2009).

Supporting this mechanism, intra-accumbens inhibition of either cystine-glutamate exchange or mGluR2/3 blocks the ability of acute NAC to reduce cocaine reinstatement (Moran et al. 2005; Kau et al. 2008). In addition, although higher concentrations of NAC (50 $\mu \mathrm{M})$ acutely applied to accumbens tissue slices promote EPSC amplitude via mGluR5 stimulation (see above), at lower concentrations ( $0.5 \mu \mathrm{M})$, NAC elicits an mGluR2/3-dependent inhibition of EPSCs, supporting the capacity of acute NAC to act via stimulating mGluR2/3 (Kupchik et al. 2012). Thus, NAC would appear to regulate cocaine reinstatement acutely by increasing cystine-glutamate exchange and indirectly activating mGluR2/3; however, it is the restoration of GLT-1 that is most critical for producing the more enduring protection from cocaine reinstatement elicited by chronic NAC treatment. 
Results presented here support a critical role for drug-induced downregulation of accumbens GLT-1 in the vulnerability to relapse, and that the restoration of GLT-1 by daily NAC treatments is a critical mechanism whereby NAC inhibits reinstated cocaine seeking. Importantly, while a number of studies demonstrate pre-clinical and clinical efficacy of NAC against cocaine seeking, results are not unilaterally positive (Mardikian et al. 2007; Berk et al. 2013). For example, in a recent double-blind placebo-controlled study of NAC for cocaine dependence over 8 weeks for outpatient, treatment-seeking users, overall use was not statistically different between groups (LaRowe et al. 2013). Importantly, however, a significant difference was found between subjects receiving NAC versus placebo control for subjects abstinent from cocaine at the outset of the study. This group may be considered analogous to the experimental design described in the current study, in which NAC is given prior to the last 5 days of the extinction training phase, hence providing a candidate cellular mechanism for the observed clinical effect. Given that a consequence of downregulated GLT-1 is increased spillover of synaptically released glutamate onto extracellular glutamate receptors (Bergles, Diamond \& Jahr 1999), these data support a strategy for treating cocaine addiction that may involve not only refining drug treatments that promote GLT-1, but also treatments that inhibit extrasynaptic glutamate receptors such as mGluR5.

\section{Acknowledgments}

The authors thank the members of the Kalivas laboratory for comments on a previous version of this manuscript. This work was supported by K99-DA026254 (K.J.R.) and R01-DA015369/DA003906 (P.W.K.).

\section{References}

Backstrom P, Hyytia P. Involvement of AMPA/kainate, NMDA, and mGlu5 receptors in the nucleus accumbens core in cue-induced reinstatement of cocaine seeking in rats. Psychopharmacology (Berl). 2007; 192:571-580. [PubMed: 17347848]

Baker DA, McFarland K, Lake RW, Shen H, Tang XC, Toda S, Kalivas PW. Neuroadaptations in cystine-glutamate exchange underlie cocaine relapse. Nat Neurosci. 2003; 6:743-749. [PubMed: 12778052]

Bergles DE, Diamond JS, Jahr CE. Clearance of glutamate inside the synapse and beyond. Curr Opin Neurobiol. 1999; 9:293-298. [PubMed: 10395570]

Berk M, Malhi GS, Gray LJ, Dean OM. The promise of N-acetylcysteine in neuropsychiatry. Trends Pharmacol Sci. 2013; 34:167-177. [PubMed: 23369637]

Bridges R, Lutgen V, Lobner D, Baker DA. Thinking outside the cleft to understand synaptic activity: contribution of the cystine-glutamate antiporter (System xc-) to normal and pathological glutamatergic signaling. Pharmacol Rev. 2012; 64:780-802. [PubMed: 22759795]

Danbolt NC. Glutamate uptake. Prog Neurobiol. 2001; 65:1-105. [PubMed: 11369436]

Dean O, Giorlando F, Berk M. N-acetylcysteine in psychiatry: current therapeutic evidence and potential mechanisms of action. J Psychiatry Neurosci. 2011; 36:78-86. [PubMed: 21118657]

Fischer-Smith KD, Houston AC, Rebec GV. Differential effects of cocaine access and withdrawal on glutamate type 1 transporter expression in rat nucleus accumbens core and shell. Neuroscience. 2012; 210:333-339. [PubMed: 22433294]

Flanagan RJ, Meredith TJ. Use of N-acetylcysteine in clinical toxicology. Am J Med. 1991; 91:131S139S. [PubMed: 1928204]

Gipson CD, Reissner KJ, Kupchik YM, Smith AC, Stankeviciute N, Hensley-Simon ME, Kalivas PW. Reinstatement of nicotine seeking is mediated by glutamatergic plasticity. Proc Natl Acad Sci USA. 2013; 110:9124-9129. [PubMed: 23671067] 
Gray KM, Carpenter MJ, Baker NL, DeSantis SM, Kryway E, Hartwell K, McRae-Clark AL, Brady KT. A double-blind randomized controlled trial of $\mathrm{N}$-acetylcysteine in cannabis-dependent adolescents. Am J Psychiatry. 2012; 169:805-812. [PubMed: 22706327]

Kalivas PW. The glutamate homeostasis hypothesis of addiction. Nat Rev Neurosci. 2009; 10:561572. [PubMed: 19571793]

Kalivas PW, Volkow ND. New medications for drug addiction hiding in glutamatergic neuroplasticity. Mol Psychiatry. 2011; 16:974-986. [PubMed: 21519339]

Kau KS, Madayag A, Mantsch JR, Grier MD, Abdulhameed O, Baker DA. Blunted cystine-glutamate antiporter function in the nucleus accumbens promotes cocaine-induced drug seeking. Neuroscience. 2008; 155:530-537. [PubMed: 18601982]

Knackstedt LA, Melendez RI, Kalivas PW. Ceftriaxone restores glutamate homeostasis and prevents relapse to cocaine seeking. Biol Psychiatry. 2010; 67:81-84. [PubMed: 19717140]

Kumaresan V, Yuan M, Yee J, Famous KR, Anderson SM, Schmidt HD, Pierce RC. Metabotropic glutamate receptor 5 (mGluR5) antagonists attenuate cocaine priming- and cue-induced reinstatement of cocaine seeking. Behav Brain Res. 2009; 202:238-244. [PubMed: 19463707]

Kupchik YM, Moussawi K, Tang XC, Wang X, Kalivas BC, Kolokithas R, Ogburn KB, Kalivas PW. The effect of $\mathrm{N}$-acetylcysteine in the nucleus accumbens on neuro-transmission and relapse to cocaine. Biol Psychiatry. 2012; 71:978-986. [PubMed: 22137594]

LaRowe SD, Kalivas PW, Nicholas JS, Randall PK, Mardikian PN, Malcolm RJ. A double-blind placebo-controlled trial of $\mathrm{N}$-acetylcysteine in the treatment of cocaine dependence. Am J Addict. 2013; 22:443-452. [PubMed: 23952889]

Lewerenz J, Hewett SJ, Huang Y, Lambros M, Gout PW, Kalivas PW, Massie A, Smolders I, Methner A, Pergande M, Smith SB, Ganapathy V, Maher P. The cystine/glutamate antiporter system x(c) $(-)$ in health and disease: from molecular mechanisms to novel therapeutic opportunities. Antioxid Redox Signal. 2013; 18:522-555. [PubMed: 22667998]

Mardikian PN, LaRowe SD, Hedden S, Kalivas PW, Malcolm RJ. An open-label trial of Nacetylcysteine for the treatment of cocaine dependence: a pilot study. Prog Neuropsychopharmacol Biol Psychiatry. 2007; 31:389-394. [PubMed: 17113207]

Martin-Fardon R, Baptista MA, Dayas CV, Weiss F. Dissociation of the effects of MTEP \{3-[(2methyl-1,3-thiazol-4-yl)ethynyl]piperidine \} on conditioned reinstatement and reinforcement: comparison between cocaine and a conventional reinforcer. J Pharmacol Exp Ther. 2009; 329:1084-1090. [PubMed: 19258516]

Melendez RI, Vuthiganon J, Kalivas PW. Regulation of extracellular glutamate in the prefrontal cortex: focus on the cystine glutamate exchanger and group I metabotropic glutamate receptors. J Pharmacol Exp Ther. 2005; 314:139-147. [PubMed: 15769865]

Millea PJ. N-acetylcysteine: multiple clinical applications. Am Fam Physician. 2009; 80:265-269. [PubMed: 19621836]

Moran MM, McFarland K, Melendez RI, Kalivas PW, Seamans JK. Cystine/glutamate exchange regulates metabotropic glutamate receptor presynaptic inhibition of excitatory transmission and vulnerability to cocaine seeking. J Neurosci. 2005; 25:6389-6393. [PubMed: 16000629]

Moussawi K, Pacchioni A, Moran M, Olive MF, Gass JT, Lavin A, Kalivas PW. N-Acetylcysteine reverses cocaine-induced metaplasticity. Nat Neurosci. 2009; 12:182-189. [PubMed: 19136971]

Moussawi K, Zhou W, Shen H, Reichel CM, See RE, Carr DB, Kalivas PW. Reversing cocaineinduced synaptic potentiation provides enduring protection from relapse. Proc Natl Acad Sci USA. 2011; 108:385-390. [PubMed: 21173236]

Olive MF, Cleva RM, Kalivas PW, Malcolm RJ. Glutamatergic medications for the treatment of drug and behavioral addictions. Pharmacol Biochem Behav. 2012; 100:801-810. [PubMed: 21536062]

Paxinos, G.; Watson, C. The Rat Brain in Stereotaxic Coordinates. 5. Amsterdam: Elsevier Academic; 2005.

Ramirez-Nino AM, D’Souza MS, Markou A. N-acetylcysteine decreased nicotine self-administration and cue-induced reinstatement of nicotine seeking in rats: comparison with the effects of $\mathrm{N}$ acetylcysteine on food responding and food seeking. Psychopharmacology (Berl). 2013; 225:473482. [PubMed: 22903390] 
Reissner KJ, Uys JD, Schwacke JH, Comte-Walters S, Rutherford-Bethard JL, Dunn TE, Blumer JB, Schey KL, Kalivas PW. AKAP signaling in reinstated cocaine seeking revealed by iTRAQ proteomic analysis. J Neurosci. 2011; 31:5648-5658. [PubMed: 21490206]

Reissner KJ, Sartor GC, Vazey EM, Dunn TE, Aston-Jones G, Kalivas PW. Use of vivo-morpholinos for control of protein expression in the adult rat brain. J Neurosci Methods. 2012; 203:354-360. [PubMed: 22027492]

Reissner KJ, Brown RM, Spencer S, Tran PK, Thomas CA, Kalivas PW. Chronic administration of the methylxanthine propentofylline impairs reinstatement to cocaine by a GLT-1 dependent mechanism. Neuropsychopharmacology. 2013; 39:499-506. [PubMed: 23985782]

Sari Y, Sreemantula SN. Neuroimmunophilin GPI-1046 reduces ethanol consumption in part through activation of GLT1 in alcohol-preferring rats. Neuroscience. 2012; 227:327-335. [PubMed: 23059796]

Sari Y, Smith KD, Ali PK, Rebec GV. Upregulation of GLT1 attenuates cue-induced reinstatement of cocaine-seeking behavior in rats. J Neurosci. 2009; 29:9239-9243. [PubMed: 19625514]

Sari Y, Sakai M, Weedman JM, Rebec GV, Bell RL. Ceftriaxone, a beta-lactam antibiotic, reduces ethanol consumption in alcohol-preferring rats. Alcohol Alcohol. 2011; 46:239-246. [PubMed: 21422004]

Schmaal L, Veltman DJ, Nederveen A, van den Brink W, Goudriaan AE. N-acetylcysteine normalizes glutamate levels in cocaine-dependent patients: a randomized crossover magnetic resonance spectroscopy study. Neuropsychopharmacology. 2012; 37:2143-2152. [PubMed: 22549117]

Trantham-Davidson H, LaLumiere RT, Reissner KJ, Kalivas PW, Knackstedt LA. Ceftriaxone normalizes nucleus accumbens synaptic transmission, glutamate transport, and export following cocaine self-administration and extinction training. J Neurosci. 2012; 32:12406-12410. [PubMed: 22956831]

Wang X, Moussawi K, Knackstedt L, Shen H, Kalivas PW. Role of mGluR5 neurotransmission in reinstated cocaine-seeking. Addict Biol. 2013; 18:40-49. [PubMed: 22340009]

Wolf ME, Ferrario CR. AMPA receptor plasticity in the nucleus accumbens after repeated exposure to cocaine. Neurosci Biobehav Rev. 2011; 35:185-211. [PubMed: 20109488]

Zhou W, Kalivas PW. N-Acetylcysteine reduces extinction responding and induces enduring reductions in cue- and heroin-induced drug-seeking. Biol Psychiatry. 2007; 63:338-340. [PubMed: 17719565] 


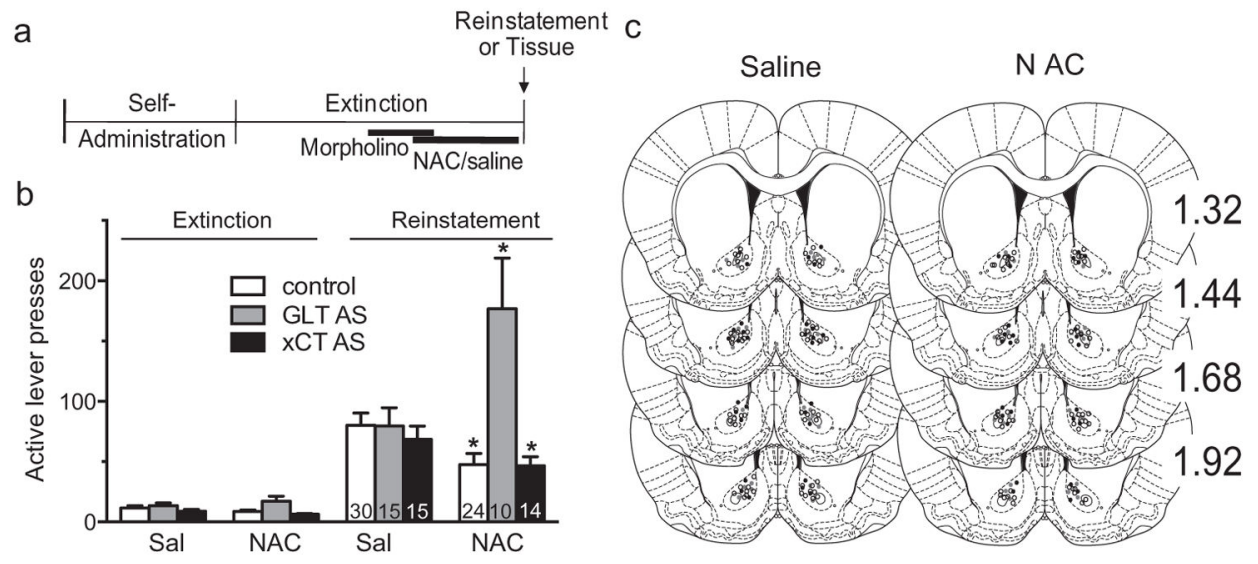

Figure 1.

Vivo-morpholino antisense inhibition of GLT-1, not xCT prevented N-acetylcysteine inhibition of cue-induced reinstatement of cocaine seeking. (a) Treatment protocol for all experiments showing the self-administration of cocaine or yoked-saline, followed by extinction and daily morpholino and NAC or saline administration that was completed 24 hours prior to either a conducting a cue-induced reinstatement session or obtaining nucleus accumbens tissue. (b) Active lever pressing during cue-induced reinstatement (120 minutes sessions) in rats extinguished from cocaine self-administration. AS-antisense vivomorpholino, Control refers to the combined GLT-1 and xCT control sequence vivomorpholino (Reissner et al. 2012). $\mathrm{N}$ is shown in bars. (c) Histologically determined location of vivo-morpholino microinjections, largely localized to the core subcompartment of the nucleus accumbens $* P<0.05$, comparing all treatment groups to Saline-Control using a two-way ANOVA followed by a Dunnett's post hoc. 
a
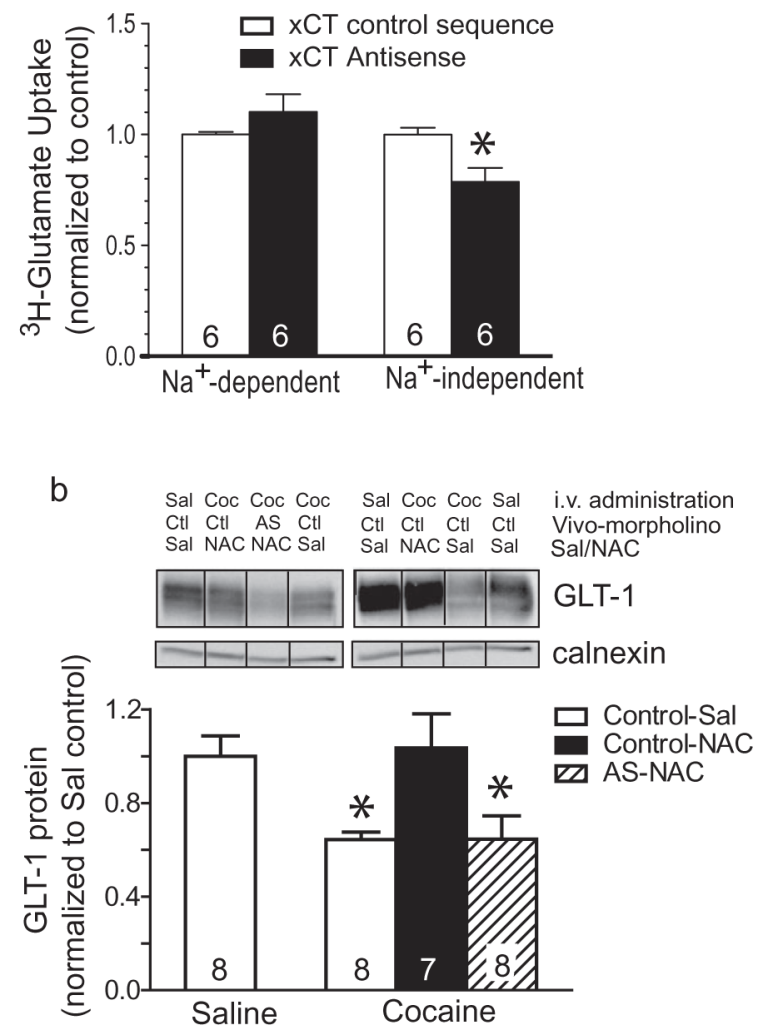

Figure 2.

Vivo-morpholino reduced xCT function and GLT-1 membrane content. (a) Rats treated into the accumbens with $\mathrm{xCT}$ vivo-morpholino antisense (AS) had reduced $\mathrm{Na}+$-independent ${ }^{3} \mathrm{H}$ glutamate uptake in accumbens tissue slices. (b) Treatment with GLT-1 vivo-morpholino AS reversed NAC-induced restoration of GLT-1 protein levels in the accumbens of rats trained to self-administer cocaine versus yoked-saline controls. $\mathrm{N}$ is shown in bars. Abcissa indicates i.v. administration group (saline or cocaine). Vivo-morpholino group and saline versus NAC treatment is indicated in the legend $* P<0.05$ compared with yoked saline using a one-way ANOVA followed by a Dunnett's post hoc comparison. 


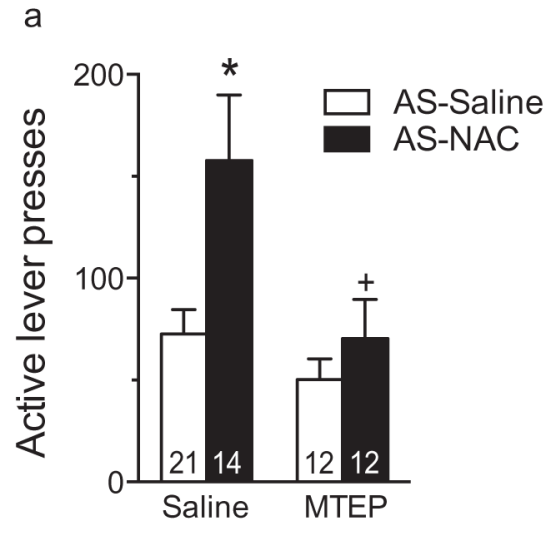

b

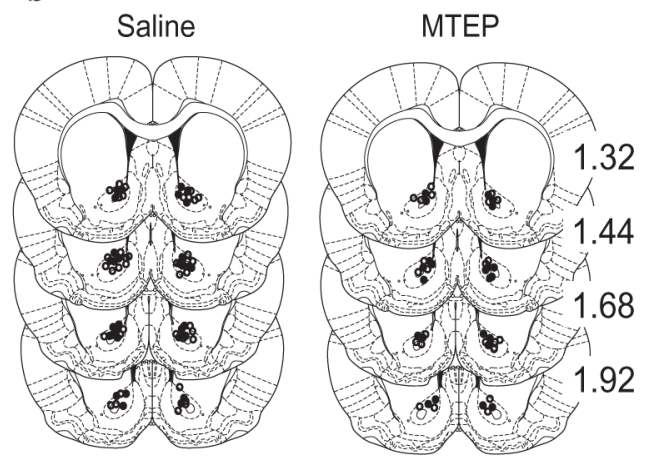

Figure 3.

Inhibiting mGluR5 prevented the augmented reinstatement elicited by NAC treatment in rats pre-treated with GLT-1 vivo-morpholino antisense.The same protocol outlined in Fig. 1a was used. All animals were treated with GLT-1 vivo-morpholino anti-sense, followed by either NAC or saline, followed by an acute administration of MTEP $(0.5 \mathrm{mg} / \mathrm{kg}$, ip) or saline (ip), 10 minutes prior to initiating cue-induced reinstatement

${ }^{*} P<0.05$, comparing NAC + saline to Saline + Saline using a Student's $t$-test followed by a Bonferroni adjustment for multiple comparisons.

$+P<0.05$, comparing NAC + saline to NAC + MTEP. 